\section{Perianale streptogene Dermatitis: Penicillin oder Cephalosporin?}

Nach einer aktuellen Studie kann eine Cephalosporin-Therapie die Rezidivrate bei der perianalen streptogenen Dermatitis im Vergleich zur Behandlung mit Penicillin signifikant reduzieren

D ie perianale streptogene Dermatitis (PSD) ist klinisch durch ein scharf begrenztes, nur gering schuppendes Erythem unterschiedlicher Intensität in der Analfalte gekennzeichnet. Der klinische Verdacht wird durch den Nachweis von $\beta$-hämolysierenden Streptokokken mittels Schnelltest oder Kultur bestätigt.

Eine orale Antibiotikabehandlung ist die Therapie der Wahl. Jedoch auch nach einer erfolgreichen Antibiotikabehandlung ist die Rezidivrate hoch.

In einer 81 Kinder mit PSD umfassenden Studie, die klinikeigene retrospektiv erhobene Daten wie auch Daten einer Metaanalyse umfasste, wurden die Beeinflussung der Rezidivrate unter Behandlung mit Penicillin bzw. Amoxicillin im Vergleich zur Behandlung mit einem $\beta$-Laktamase-stabilen Antibiotikum (überwiegend Cephalosporine) untersucht [1].

Die Untersuchung belegte die aus der Literatur bekannte hohe Rezidivquote, die bei $32,1 \%$ lag (26/81), wobei $69,2 \%$ der Kinder (18/26) innerhalb der ersten sechs Wochen nach Behandlung ein PDSRezidiv entwickelten. Jedoch unterschied sich die Rezidivquote in Abhängigkeit vom verwendeten Antibiotikum signifikant: einer Rezidivrate von 38,1\% (16/42) unter der Penicillin/Amoxicillin-Behandlung stand eine Rezidivrate von 27,8\% (10/36) unter der Behandlung mit einem $\beta$-Laktamase-stabilen Antibiotikum gegenüber (angepasste Odds Ratio: $2,02)$.

Fazit: Diese Arbeit bestätigt die Ergebnisse einer früheren Untersuchung zur Therapie der PDS, in der Cefuroxim der
Perianale streptogene Dermatitis: scharf begrenztes Erythem im Bereich der Analfalte mit fibrinösen Auflagerungen

klassischen Penicillin-Behandlung hinsichtlich dem klinischem Ansprechen und der Erregereradikation signifikant überlegen war [2]. Die Autoren der vorliegenden Studie diskutieren als Grund für die bessere Wirksamkeit der CefuroximTherapie, dass durch die Verwendung eines $\beta$-Laktamase stabilen Antibiotikums auch weitere Erreger im Analbereich eliminiert werden, die sonst eine Wirksamkeit von Penicillin reduziert hätten.

Prof. Dr. Dietrich Abeck

1. Olson D, Edmondson MB. Outcomes in children treated for perineal group $A$ beta-hemolytic streptococcal dermatitis. Pediatr Infect Dis 2011; 30: 933-6

2. Meury SN et al. Randomized, comparative efficacy trial of oral penicicillin versus cefuroxime for perianal streptococcal dermatitis in children.

J Pediatr 2008; 153: 799-802

\title{
Vitamin D bei hohem Hautkrebsrisiko?
}

\section{Ob Vitamin D die Entstehung von Melanomen und Nicht-Melanom- Hautkrebs (NMSC) verhindern kann, ist bislang unklar. US-Kollegen nutzten die „Women's Health Initiative“, um dieser Frage nachzugehen.}

$\mathrm{D}$ as mögliche Potenzial von Vitamin D, Hautkrebs zu verhindern, wird schon seit längerem diskutiert. So entwickeln Mäuse, denen der Vitamin-D-Rezeptor fehlt, besonders häufig ein NMSC, und die Behandlung mit Vitamin D kann in vitro und auch in Mausmodellen das Wachsum von NMSC und Melanomzellen hemmen. Auch beim Menschen gibt es Hinweise für einen Schutz durch Vitamin D: So scheinen höhere Serumspiegel an 25-Hydroxyvitamin D sowohl mit einem reduzierten NMSC-Risiko verbunden zu sein als auch mit einem längeren Überleben nach einer Melanom-Diagnose. Randomisierte klinische Studien zu diesem Thema wurden bisher aber nicht durchgeführt. US-amerikanische Autoren der vorliegenden Arbeit nutzten deshalb eine randomisierte, placebokontrollierte Studie im Rahmen der „Women's Health Initiative", bei der eigentlich der Einfluss von Kalzium und Vitamin D auf die Entwicklung von Frakturen und Kolonkarzinom bei postmenopausalen Frauen untersucht wurde.

36.282 Frauen zwischen 50 und 79 Jahren erhielten nach Randomisierung sieben Jahre lang täglich entweder $1.000 \mathrm{mg}$ Kalzium plus 400 IU Vitamin D3 (CaD) oder ein Placebo. Jedes Jahr wurden die Frauen nach dem Auftreten von Hautkrebs befragt. Insgesamt unterschied sich weder die Zahl der NMSC-Fälle noch die der Melanomfälle zwischen der $\mathrm{CaD}$ - und der Placebo-Gruppe (Hazard Ratio, HR: 1,02 für NMSC bzw. 0,86 für Melanom). Subgruppenanalysen ergaben jedoch, dass
Frauen mit einer NMSC-Anamnese womöglich doch von Kalzium plus Vitamin D profitieren: Ihr Risiko lag im Vergleich $\mathrm{zu}$ jenen, die ein Placebo erhielten, signifikant geringer (HR: 0,43, $\mathrm{p}=0,038$ ).

Fazit: Eine relativ geringe Dosis von Kalzium und Vitamin D konnte die Gesamtinzidenz von NMSC oder Melanom bei postmenopausalen Frauen nicht senken. Für diejenigen Frauen, bei denen früher schon einmal ein NMSC aufgetreten war, konnte allerdings ein Anti-MelanomEffekt nachgewiesen werden. Wie jede retrospektive Subgruppenanalyse ist aber auch diese mit Vorsicht zu genießen, möglicherweise profitieren Frauen mit hohem Hautkrebsrisiko jedoch von der Supplementierung.

Christina Berndt

Tang JY et al. Calcium plus vitamin D supplementation and the risk of nonmelanoma and melanoma skin cancer: Post hoc analyses of the Women's Health Initiative randomized controlled trial.

J Clin Oncol. 2011; 29: 3078-84 